\title{
Polarization of $\Lambda$-hyperons, vorticity and helicity structure in heavy-ion collisions
}

\author{
Aleksei Zinchenko ${ }^{a},{ }^{*}$ Oleg Teryaev ${ }^{b}$, Mircea Baznat ${ }^{b, c}$ and Alexander Sorin ${ }^{b}$ \\ ${ }^{a}$ Department of Theoretical Physics, P. G. Demidov Yaroslavl State University, \\ Sovietskaya 14, 150003 Yaroslavl, Russia \\ ${ }^{b}$ Joint Institute for Nuclear Research, Joliot-Curie 6, 141980 Dubna, Russia \\ ${ }^{c}$ Institute of Applied Physics, Academy of Sciences of Moldova, \\ Academiei 5, MD-2028 Chisinau, Moldova \\ E-mail: a.zinchenko1@uniyar.ac.ru, teryaev@theor.jinr.ru
}

Simulations of peripheral Au+Au collisions at NICA energies are performed in the PHSD transport model. The properties of velocity and vorticity fields as well as hydrodynamic helicity are studied at different impact parameters and energies. A general structure of the velocity field follows the "little bang" pattern which may be quantified by the velocity dependence allowing to extract the "Hubble" constant. A quadrupole-like structure of the vorticity field in all the planes is obtained. The helicity-separation effect is found. Calculations of the $\Lambda$-hyperon polarization is performed within the thermodynamic and anomalous models at NICA energies.

\footnotetext{
*** The European Physical Society Conference on High Energy Physics (EPS-HEP2021), *** ***26-30 July $2021 * * *$

*** Online conference, jointly organized by Universität Hamburg and the research center DESY ***
}

\footnotetext{
${ }^{*}$ Speaker
} 


\section{Introduction}

Experimental data obtained by the STAR collaboration [1] indicate a polarization of hyperons produced in heavy-ion collisions. and its decrease with an energy increase. Theoretical analysis [2] suggests that the $\Lambda$-polarization decrease with the energy is related to a suppression of the Axial Magnetic effect contribution in strongly correlated QCD matter. This approach (although related [3]) is based on the vortical effect (see e. g. [4]) that, being the macroscopic manifestation of the axial anomaly [5], leads to an induced axial current of strange quarks which may be converted to the $\Lambda$-hyperon polarization $[6,7]$. This polarization is proportional to the strange axial charge $Q_{5}^{s}$ :

$$
\left\langle\Pi_{0}^{\Lambda}\right\rangle=\left\langle\frac{m_{\Lambda}}{N_{\Lambda} p_{y}}\right\rangle Q_{5}^{s}=\left\langle\frac{m_{\Lambda}}{N_{\Lambda} p_{y}}\right\rangle \frac{N_{c}}{2 \pi^{2}} \int d^{3} x \mu_{s}^{2}(\vec{x}) \gamma^{2}(t, \vec{x}) \epsilon^{i j k} v_{i}(t, \vec{x}) \partial_{j} v_{k}(t, \vec{x})
$$

where $m_{\Lambda}$ is the mass of the $\Lambda$-hyperon, $N_{\Lambda}$ is their number, $N_{c}$ is the number of quark colors. The relativistic definition of the kinetic vorticity is used: $\omega_{\mu v}=\left(\partial_{\nu} u_{\mu}-\partial_{\mu} u_{\nu}\right) / 2$, with $u_{v}(t, \vec{x})=\gamma(t, \vec{x})\{1, \vec{v}(t, \vec{x})\}$ being a relativistic four-velocity field and $\gamma(t, \vec{x})=1 / \sqrt{1-v^{2}(t, \vec{x})}$. The hydrodynamical helicity, $H \equiv \int d^{3} x(\vec{v} \cdot \vec{\omega})$, depends on the velocity and vorticity and implicitly affect the polarization, so it is important to know structures of both these fields.

\section{Vorticity structure}

Nuclear collisions are simulated in the PHSD transport model [8]. All data are transferred to a larger grid with averaging over all parameters with statistics about $10^{6}$ events. The cell velocity is defined as follows:

$$
v_{1}^{a}(x)=\frac{\sum_{i} p_{i}^{a} F\left(x, x_{i}\right)}{\sum_{i} p_{i}^{0} F\left(x, x_{i}\right)},
$$

where $F\left(x, x_{i}\right)$ is a smearing function, $a=1,2,3$ are the spatial indices, $p_{i}^{a}$ and $p_{i}^{0}$ are the momentum and energy of the $i$-th particle, and the summation is over all the particles in a cell.

The frame is used in which nuclei collide along the $Z$-axis and the reaction plane coincides with the $X Z$-plane. An increase in the $X$-projection of the vorticity is observed around the fireball boundary, as seen in Fig. 1, and there are maxima at the boundary with spectators. One can also see a mirror quadrupole structure with an internal part having an opposite sign with respect to the corresponding external region.

The longitudinal component $\omega_{z}$ of the vorticity has the same structure in the $X Y$-plane, as seen in Fig. 2, the averaging was done over the cylinder along the $Z$-axis from -1.25 to $1.25 \mathrm{fm}$. The transverse vorticity $\vec{\omega}_{\perp}=\left(\omega_{x}, \omega_{y}\right)$ in the same plane has a symmetric structure with respect to $Y$-axis at $Z=0$ but moving away from the fireball center external and internal rotations in the opposite direction are observed as shown in Fig. 3. It is also seen that keeping the sign of $\omega_{z}$ at $Z<0$ and $Z>0$ (two bottom plots), $\vec{\omega}_{\perp}$ field rotates in different directions (two top plots). Moving away from the fireball center, the iternal rotation disappears which is seen in Fig. 4 and this structure coincides with the one in [9], where the transverse vorticity field $\vec{\omega}_{\perp}$ is constructed for the pseudorapidity $\eta_{s}=(1 / 2) \ln [(t+z) /(t-z)]= \pm 1$ at $t=9.5 \mathrm{fm} / c$. The picture differs in the reaction plane (Fig. 5) where the non-quadrupole vorticity is observed. The total vorticity 


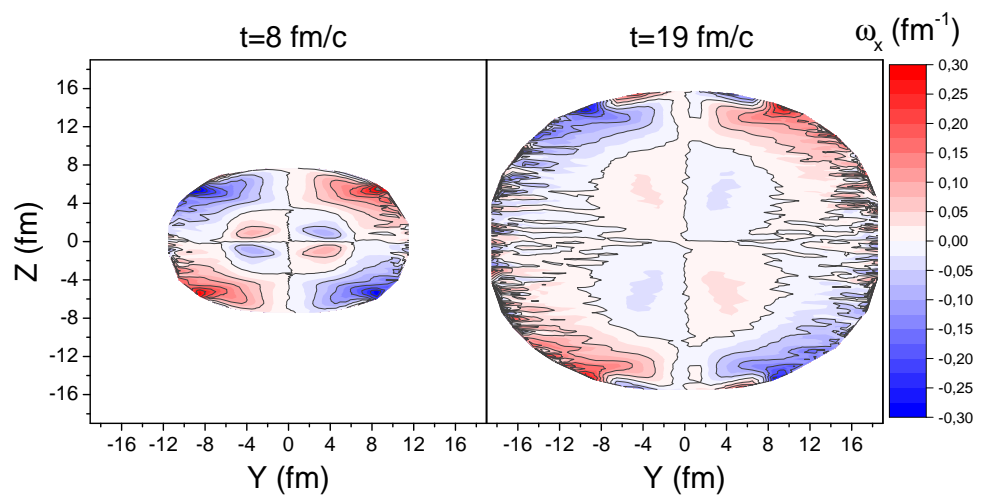

Figure 1: The quadrupole structure of the relativistic vorticity projection $\omega_{x}$ in $\mathrm{Au}+\mathrm{Au}$ at the energy $\sqrt{s}=7.7 \mathrm{GeV}, X=0$, and the impact parameter $b=7 \mathrm{fm}$.

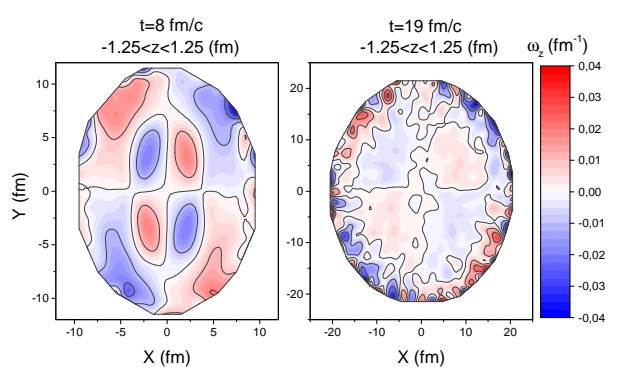

Figure 2: The quadrupole structure of $\omega_{z}$ in $\mathrm{Au}+\mathrm{Au}$ at $\sqrt{s}=7.7 \mathrm{GeV}$ at different time. Averaging is performed in the $2.5 \mathrm{fm}$ range in the layer $z=0$.

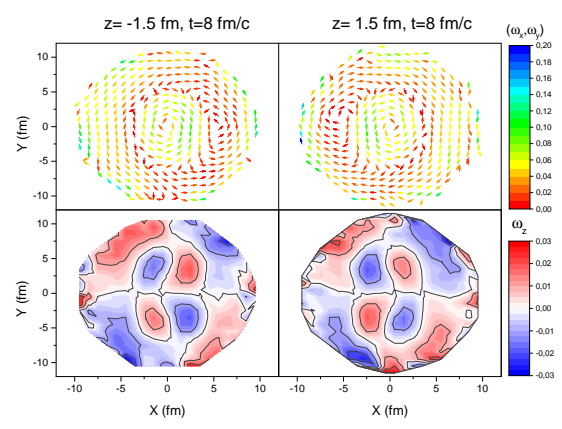

Figure 3: The quadrupole structure of $\omega_{z}$ in $\mathrm{Au}+\mathrm{Au}$ at $\sqrt{s}=7.7 \mathrm{GeV}$ (two bottom plots) and the structure of the transverse vorticity $\vec{\omega}_{\perp}=$ $\left(\omega_{x}, \omega_{y}\right)$ in the same layer of $z$ and at the same time (two top plots).

contribution in this plane is non-zero because $\omega_{y}$ has the same sign on the boundaries of the fireball and spectators, and it exceeds substantially the values in other parts of the fireball.

In conclusion, the vorticity field has a spherical elongated shape with maximum values at poles along $Z$-axis, and one half of this sphere rotates in one direction and the other in the reverse one, having the opposite rotation in the center.

\section{Helicity separation and polarization}

The helicity contributes directly to the polarization of hyperons in the so-called axial vortical effect approach, as seen from (1). The helicity separation effect was discovered in QGSM [10]. This effect is also exists in the PHSD model (Fig. 6) and receives the significant contribution $\sim v_{z} \omega_{z}$ from the longitudinal projections of the velocity and vorticity. It assumes a separation with respect to the momentum $Y$-component but there is also a spatial separation.

The polarization of $\Lambda$-hyperons is calculated using the axial anomaly approach and is about $\Pi_{0}^{\Lambda}=8 \%$ for the impact parameter $b=7 \mathrm{fm}$, energy $\sqrt{s}=7.7 \mathrm{GeV}$ and rapidity $|y|<1$, where 


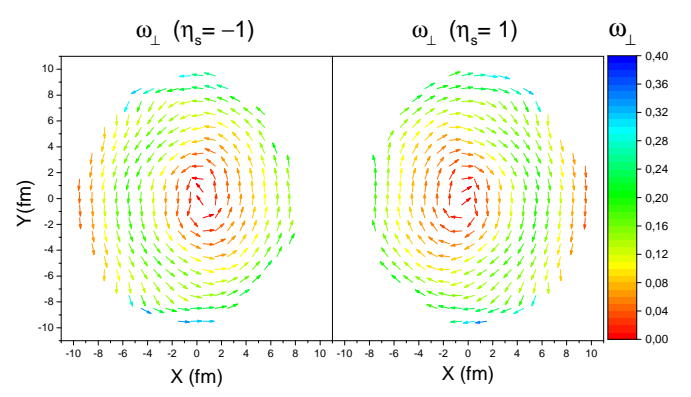

Figure 4: The distribution of the transverse vorticity $\vec{\omega}_{\perp}$ in the transverse plane at longitudinal position $\eta_{s}=-1$ (left) and $\eta_{s}=1$ (right) at the time $t=$ $9.5 \mathrm{fm} / c$ and $b=5 \mathrm{fm}$.

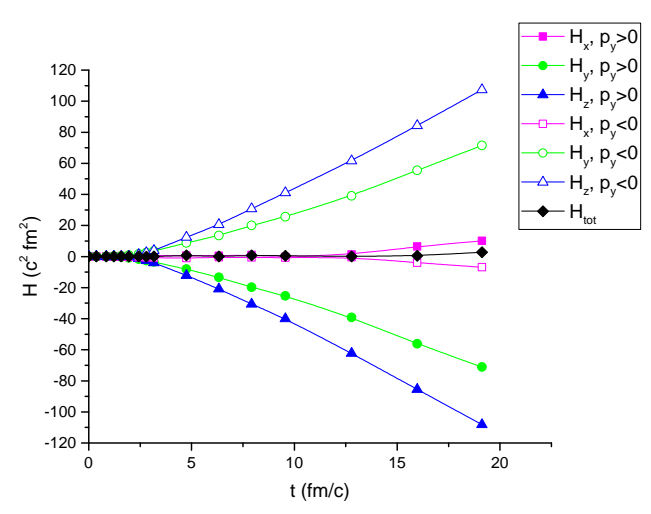

Figure 6: Separation of the helicity components relative to $y$-component of momentum at different times (impact parameter $b=7 \mathrm{fm}, \sqrt{s}=7.7 \mathrm{GeV}$ ).

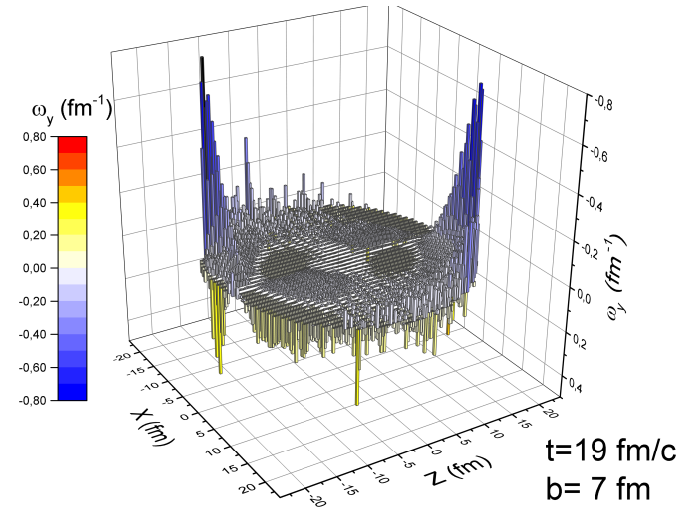

Figure 5: $Y$-component of relativistic vorticity in the reaction plane $(Y=0)$ at the energy $\sqrt{s}=7.7 \mathrm{GeV}$.

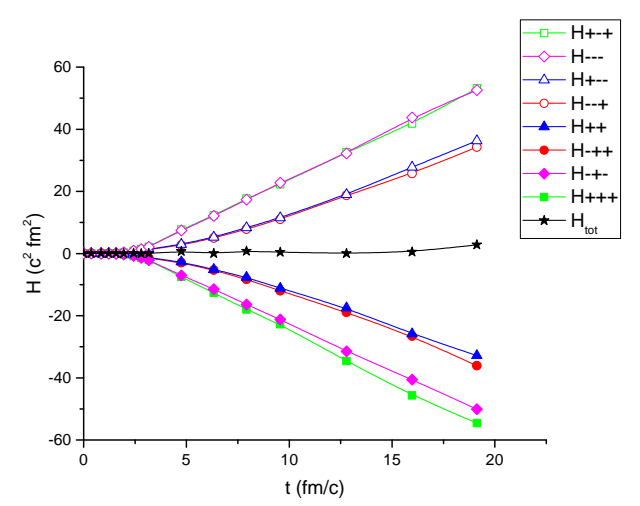

Figure 7: Helicity separation relative to spatial octants (impact parameter $b=7 \mathrm{fm}, \sqrt{s}=7.7 \mathrm{GeV}$ ), "+++" means that integration was performed in oc$\operatorname{tant} x>0, y>0, z>0$ and others accordingly.

$\left.y=(1 / 2) \ln \left[\left(E+p_{z}\right) /\left(E-p_{z}\right)\right]\right)$, and it is about $6 \%$ for centrality $20-50 \%$. The polarization calculated within the approach exploring the local equilibrium thermodynamics [11] is about $2 \%$ for centrality 20-50\%. In the thermodynamic approach, the spin $\vec{S}^{*}$ averaged over the $\vec{p}_{\Lambda}$ direction is determined by

$$
\left\langle\vec{S}_{\Lambda}^{*}\right\rangle_{\vec{n}_{p}}=\frac{1-n_{\Lambda}}{4 M_{\Lambda}}\left(E_{\Lambda}+\frac{1}{3} \frac{\vec{p}_{\Lambda}^{2}}{E_{\Lambda}+m_{\Lambda}}\right) \operatorname{rot} \vec{\beta}
$$

where $\vec{\beta}=\vec{v} / T$ is the thermodynamic velocity and $\Pi^{\Lambda} \sim 2\left\langle\vec{S}_{\Lambda}^{*}\right\rangle_{\vec{n}_{p}}$. One can see some difference which can be related, firstly, to the choice of velocity determination, secondly, to the sensitivity of thermodynamic quantities and, finally, it can be also affected by the gravitational anomaly contribution [10] suppressed due to the collective effects revealed in lattice simulations. 


\section{4. "Hubble low" in PHSD}

A linear dependence of the particle velocity on the transverse radius $\rho=\sqrt{x^{2}+y^{2}}$ in the vertical plane $(Z=0)$ is not observed near the center of the fireball because decay and scattering reactions occur at small velocity which violates an isotropy. At the large radii, particles approach the maximum velocity corresponding to the initial velocity of the colliding nuclei which prevents the linear growth.

A linear dependence of the velocity of particles in the medium area of the fireball at times exceeding $15 \mathrm{fm} / c$, which corresponds to time of chemical freezout, is seen in Fig. 8. This area can be called the "Little Bang" and the Hubble law with the constant $H$ can be defined.

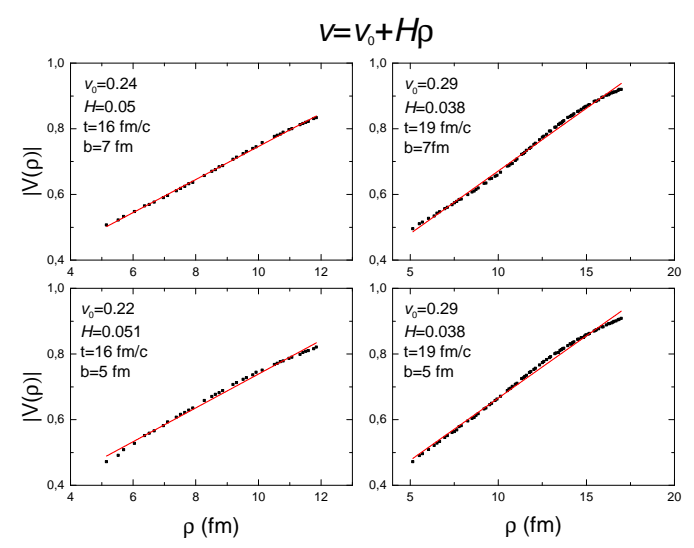

Figure 8: The dependence of particles velocity on the transverse radius $\rho=\sqrt{x^{2}+y^{2}}$ at $z=0$ as the "Hubble law" at the energy $\sqrt{s}=7.7 \mathrm{GeV}$ with $b=5 \mathrm{fm}$ and $b=7 \mathrm{fm}$.
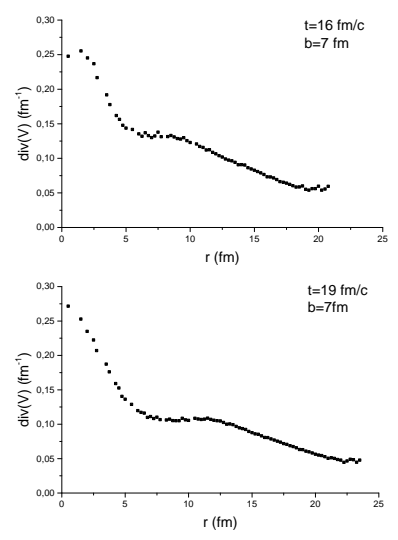

Figure 9: The dependence of the particle velocity divergence on the distance to the fireball center.

Since there is a linear dependence of the velocity on the distance to the particle, it has a sense to check the velocity divergence and to verify the isotropy of the medium in this region. As can be seen in Fig. 9, the divergence for the most part does not have the form $\operatorname{div}(v)=$ const. This suggests that in almost the entire volume the explosion is anisotropic. Only in a small area, where particles are already formed and fly apart without interaction, it is possible to determine a uniform expansion $(6<\rho<9 \mathrm{fm}$ for $t=16 \mathrm{fm} / c$ and $6.5<\rho<11.5 \mathrm{fm}$ for $t=19 \mathrm{fm} / c)$. This region corresponds to a half of the linear velocity region only. It determines the constant value of the divergence $\operatorname{div}(v)=0.14 \mathrm{fm}^{-1}$ at $t=16 \mathrm{fm} / c$ and $\operatorname{div}(v)=0.11 \mathrm{fm}^{-1}$ at $t=19 \mathrm{fm} / c$, which gives us the fulfillment of the equality $\operatorname{div}(v) \simeq 3 \mathrm{H}$.

\section{Summary}

The structure of the vorticity field is studied and similar patterns are obtained in different projections. The main structure in planes perpendicular to the reaction plane is quadrupole-like. The helicity separation effect is established in the PHSD model both in momentum and position space. A region inside the fireball is discovered, where the scattering matter of the post-collision medium obeys the Hubble law. The polarization of $\Lambda$-hyperons is calculated in two different approaches 
and turns out to be close to that found at energy $\sqrt{s}=7.7 \mathrm{GeV}$ by the STAR collaboration. The results of the thermodynamic equilibrium model are closer to the experiment $\left(\Pi_{\exp }^{\Lambda} \sim 2 \%\right)$, which is explained by the lower sensitivity to thermodynamic quantities.

\section{Acknowledgements}

We are grateful to E. Bratkovskaya, Yu. Ivanov, E. Kolomeitsev, V. Toneev and V. Voronyuk for useful discussions. The reported study was funded by RFBR according to the research project number 20-32-90205.

\section{References}

[1] L. Adamczyk et al. [STAR Collaboration], Nature 548, 62 (2017) [arXiv:1701.06657 [nuclex]].

[2] A. Sorin and O. Teryaev, Phys. Rev. C 95, no. 1, 011902 (2017) [arXiv:1606.08398 [nucl-th]].

[3] G. Prokhorov and O. Teryaev, arXiv:1707.02491 [hep-th].

[4] T. Kalaydzhyan, Phys. Rev. D 89, no. 10, 105012 (2014) [arXiv:1403.1256 [hep-th]].

[5] D.T. Son and P. Surowka, Phys. Rev. Lett. 103, 191601 (2009) [arXiv:0906.5044 [hep-th]].

[6] O. Rogachevsky, A. Sorin and O. Teryaev, Phys. Rev. C 82, 054910 (2010) [arXiv:1006.1331 [hep-ph]].

[7] J. -H. Gao, Z. -T. Liang, S. Pu, Q. Wang and X. -N. Wang, Phys. Rev. Lett. 109, 232301 (2012) [arXiv:1203.0725 [hep-ph]].

[8] W. Cassing and E. L. Bratkovskaya, Nucl. Phys. A 831, 215 (2009) [arXiv:0907.5331 [nuclth]].

[9] X. L. Xia, H. Li, Z. B. Tang and Q. Wang, Phys. Rev. C 98, 024905 (2018) [arXiv:1803.00867 [nucl-th]].

[10] M. Baznat, K. Gudima, A. Sorin and O. Teryaev, Phys. Rev. C 88, 061901 (2013) [arXiv:1301.7003 [nucl-th]].

[11] F. Becattini, L. Bucciantini, E. Grossi and L. Tinti, Eur. Phys. J. C 75, no. 5, 191 (2015) [arXiv:1403.6265 [hep-th]]. 